Dialectologia. Special issue, IX (2021), 39-67.

ISSN: 2013-2247

Received 19 April 2021.

Accepted 30 April 2021.

\title{
LO QUE EL ALPI LE OFRECÍA A LA LINGÜÍSTICA GALLEGA EN $1962^{1}$
}

\author{
Francisco DUBERT-GARCíA \\ Instituto da Lingua Galega / Universidade de Santiago de Compostela * \\ francisco.dubert@usc.es
}

\section{Resumen}

A principios de los años 60 del siglo XX existía una conciencia clara y generalizada sobre el profundo desconocimiento acerca del gallego popular; la mayoría de las gramáticas eran normativas y técnicamente poco eficientes. En el año 1962 apareció el primer volumen del ALPI, con datos del gallego popular recogidos a mediados de los años 30; esta publicación fue conocida y saludada por los intelectuales ligados al galleguismo; con todo, y a pesar de que los mapas publicados contenían información sobre algunos fenómenos dialectales de gran interés, este volumen prácticamente no tuvo ninguna influencia en la naciente lingüística gallega. En este trabajo procuro mostrar qué información relevante aportaba el citado primer volumen del ALPI y presento algunas hipótesis que pretenden explicar por qué esta obra fue olvidada.

\section{Palabras clave}

$A L P I$, dialectología gallega, geolingüística gallega, historiografía de la lingüística gallega, gallego

\section{THE ALPI'S CONTRIBUTION TO GALICIAN LINGUISTICS IN 1962}

\section{Abstract}

At the beginning of the 1960s, there was a clear and shared awareness concerning the profound lack of knowledge about popular Galician; most grammars were normative and technically lacking effectiveness. In 1962 the first volume of the ALPI was published, containing data on popular

\footnotetext{
${ }^{1}$ Este trabajo se realiza con la ayuda PGC2018-095077-B-C44 del MCIU. Quiero mostrar mi agradecimiento a Xulio Sousa por sus comentarios.

* Facultade de Filoloxía, Avenida Castelao s/n, 15782 Santiago de Compostela, España.
} 
Galician gathered during the mid-1930s. Despite the publication having been well-received by Galicianist intellectuals, and despite the great interest of the information about several dialectal phenomena documented in the maps, the volume had practically no influence on the budding Galician linguistics. This paper aims to show the significant information included in the first volume of the $A L P I$, as well as to present several hypotheses on why this work was forgotten.

\section{Keywords}

$A L P I$, Galician dialectology, Galician geolinguistics, history of Galician linguistics, Galician

\section{Introducción}

Es un lugar común en la historiografía lingüística gallega referirse al "estado de extrema penuria, especialmente no tocante ao coñecemento da lingua falada," (Regueira 2008: 573) en que se encontraba nuestra disciplina hasta los años 70 del siglo pasado. Es también evidente que este hecho resulta, entre otros factores de carácter social (Santamarina 2005: 192), de la falta de enseñanza de lingüística en la Universidad de Santiago de Compostela. Basta leer el testimonio de Ricardo Carvalho Calero (nacido en 1910), que nos muestra cuál era el estado, no de la lingüística gallega, sino incluso de la lingüística en general, en la Universidad de Santiago durante los años 30 del siglo XIX: aunque el uso del gallego era común en el Seminario de Estudos Galegos, ${ }^{2}$

A investigaçom lingüistica nom chegou nunca a concretar-se em plasmaçons efectivas. Nunca dispuxo o Seminário de lingüístas profissionais de formaçom especializada e de dedicaçom plena que imprimissem o impulso necessário aos estudos sobre o galego. A Universidade de Santiago, viveiro onde geralmente se formavam os membros do Seminário, nom contava com umha Faculdade nem uma Secçom de Filologia. (Carballo Calero 1981a: 111-112)

\footnotetext{
${ }^{2}$ El Seminario de Estudos Galegos fue una institución de investigación creada en 1923 por intelectuales galleguistas con la finalidad de estudiar el patrimonio cultural gallego, labor que la Universidad de Santiago de Compostela no realizaba.
} 
En cuanto a su formación académica, Carvalho Calero indica que Armando Cotarelo Valledor, ${ }^{3}$ antes de trasladarse a Madrid, "proporcionou algumha base científico-filológica e lingüística às primeiras promoçons do Seminário" por medio de unas "leiçons de fonética histórica comparada do castelam e do galego" en el curso preparatorio de Derecho, "mais passado este curso, continuavam os estudos desta Facultade emarcados dentro da secçom de História, de jeito que havia pouca ocasiom de consolidar umha iniciaçom na lingüística". La llegada de Abelardo Moralejo como catedrático de latín permitió aprender bien esta lengua, pero "uma formaçom completa de lingüísta nom se podia adquirir em Santiago", sino que había que desplazarse a Madrid o a Salamanca (Carballo Calero 1981a: 112-113 passim). De hecho, Aníbal Otero (nacido en 1911), el investigador gallego implicado en el Atlas Lingüístico de la Península Ibérica $(A L P I)$, se había formado en la Universidad Central de Madrid, donde ingresó en 1926; en 1931 entró a trabajar en el Centro de Estudios Históricos. Según Santamarina (1975: 7), “ó principiaren os traballos do ALPI pasóu a formar parte do equipo encargado de facé-lo labor de campo" (véase también Sousa 2008: 303). Más adelante, investigadores gallegos como José Luis Pensado Tomé (nacido en 1924), Pilar Vázquez Cuesta (n. 1926), Xesús Alonso Montero (n. 1928), Amable Veiga (n. 1929), Ramón Lorenzo (n. 1935) o Xosé Luís Méndez Ferrín (n. 1938) también tuvieron que recibir su formación en Madrid. Esta situación cambiará cuando se cree en Universidad de Santiago la sección de Filología Románica en 1963 y se comiencen a impartir clases de lengua gallega en 1965, por parte, precisamente, de Carvalho Calero (Regueira 1996: 56).

No deja de ser aleccionador el hecho de que las afirmaciones de Carvalho Calero sirvan para los años 30 del siglo XX, durante los cuales Aníbal Otero, Lorenzo Rodríguez Castellano y Aurelio M. Espinosa completan la recolección de los materiales gallegos del $A L P I{ }^{4}$ el propio trabajo de recolección fue ignorado en la Galicia del momento, a

\footnotetext{
${ }^{3}$ Catedrático de lengua y literatura españolas en la Universidad de Santiago de Compostela. En 1925 se trasladó a Madrid: fue numerario de la Real Academia Española, miembro de la Real Academia de la Historia y catedrático de Filología Galaico-Portuguesa en la Universidad Central.

${ }^{4}$ En 1936 estaban completos los datos del gallego, pues al inicio de la Guerra Civil ya estaban realizadas las encuestas de Asturias, León, Zamora y Galicia (Sanchis Guaner et al. 1961: 114); más datos en Sousa (2008: 303-304). Aníbal Otero fue arrestado mientras realizaba entrevistas en territorio portugués (Alonso Montero 2009; Sousa 2008).
} 
tal punto que, cuando se tiene noticia de la reanudación de los trabajos del ALPI tras la Guerra Civil, la propia Real Academia Galega (RAG) se dirige al CSIC el 2 de octubre de 1952 solicitando que también se estudie el territorio gallego, que "no sea olvidada la región gallega, poblada por dos millones y medio de habitantes que, aun hoy, hablan en su mayoría el lenguaje regional" (apud Alonso Montero 2009: 16; véase también Sousa, en prensa 1). Es claro que en 1952 los académicos de la RAG (ese "Cuerpo Literario", como se definen) desconocían el trabajo realizado veinte años antes, lo que es comprensible, pues las catástrofes sucesivas de la Guerra Civil y la dictadura habían desmantelado el proyecto, destruido el equipo de trabajo e impedido la publicación de los resultados.

Como se sabe, en 1962 se publica el primer y único volumen del ALPI (de ahora en adelante, ALPI 1962). Este volumen contiene 75 mapas, de los cuales solo 70 permiten una verdadera labor dialectal comparativa, pues los mapas 1 y 2 contienen los nombres de los lugares; el 3, la denominación de los habitantes; el 4, los límites entre las lenguas y los nombres del habla local; y el mapa 5 muestra los nombres de los responsables de las encuestas. Con todo, veremos que los 70 que permiten realizar las comparaciones interdialectales aportan una interesante cantidad de información sobre el gallego que, curiosamente, también pasó casi desatendida para los investigadores gallegos que estaban inaugurando la tradición contemporánea de estudio lingüístico del gallego.

Este trabajo tiene dos propósitos: el primero, señalar una serie de hipótesis que busquen explicar a qué se debe ese olvido del ALPI 1962 en una época en que era notoria y reconocida la falta de datos sobre el gallego dialectal; el segundo, mostrar qué tipo y cantidad de información dialectal ofrecía el ALPI 1962. Para ello, en §2 muestro que la publicación del ALPI 1962 no fue desconocida en Galicia; en §3 presento una serie de hipótesis sobre las razones del olvido de esta obra en la dialectología gallega; en $\S 4$ la información que contiene el volumen; finalmente, en $\S 5$, van unas pequeñas conclusiones. 


\section{Conocimiento y olvido de los trabajos del ALPI 1962}

Dije arriba que la información que aportaba el ALPI 1962 pasó desatendida en la lingüística gallega posterior, a pesar de que, como voy a mostrar, su publicación fue bien conocida (y mismo saludada) en los ámbitos culturales y académicos de nuestro país.

En el ámbito cultural, en primer lugar, Xesús Alonso Montero publicó tres reportajes en diferentes periódicos bien conocidos de Galicia; ${ }^{5}$ en uno de estos trabajos, Alonso Montero reflexiona incluso sobre cómo los datos del ALPI 1962 iluminan los proyectos de normativización del gallego, una de las grandes preocupaciones intelectuales sobre la lengua gallega en aquella época. En segundo lugar, en 1963, Alonso Montero y Epifanio Ramos realizaron la exposición Cen anos de literatura galega, en la que se dedicó un apartado dedicado a Aníbal Otero. En tercer lugar, el 24 de diciembre de 1963 se propone la entrada de Aníbal Otero en la RAG como académico de número; en la propuesta, los académicos declaraban que Otero, "colaborador do Atlas Lingüístico de la Península Ibérica", era "entre todolos galegos aitualmente vivos, o máis profundo e amplio conocedor da nosa realidade idiomática ademáis de ser o investigador que máis traballos científicos leva aportado á lingüística galega nos derradeiros 20 anos" (para todo esto, Alonso Montero 2009: 19-21, passim). Estos hechos muestran que el ALPI 1962 fue una publicación conocida y valorada en el ámbito cultural.

En el mundo académico, el reconocimiento del ALPI 1962 parece ser algo más tardío. Cuatro años después de su aparición, Ricardo Carvalho Calero publica su Gramática elemental del gallego común (Carballo Calero 1966), la gramática gallega más influyente hasta mediados de los años 80 del siglo XX. Esta obra conoce varias ediciones y revisiones entre 1966 y 1979 a lo largo de las cuales Carvalho Calero va enriqueciendo paulatinamente el número de referencias de su capítulo "Orientación

\footnotetext{
${ }^{5}$ El primero, el 25 de abril de 1962 en El Progreso de Lugo ("Aníbal Otero, un filólogo lugués cuyo nombre empieza a recorrer el mundo / Es uno de los autores del Altas Lingüístico de la Península Ibérica"); el segundo, el 1 de marzo en La Noche de Santiago ("Galicia en el Atlas Lingüístico de la Península Ibérica ( Aníbal Otero, filólogo y colaborador"); y el tercero, el 3 de junio en el Faro de Vigo (“El Atlas Lingüístico de la Península Ibérica”).
} 
bibliográfica": en ningún momento introdujo el ALPI 1962 entre los títulos de dialectología.

Más llamativo es todavía el hecho de que Carvalho Calero tampoco cite el ALPI 1962 en las diferentes revisiones de sus trabajos sobre dialectología gallega (Carballo Calero 1969, 1978, 1979, 1981b). Como después veremos, no deja de ser interesante que los datos de ALPI 1962 arrojen luz sobre la distribución geográfica de algunos de los fenómenos que Carvalho Calero usa para establecer lo que denomina las modalidades del gallego: la gheada, el seseo, los tipos de fricativas coronales ${ }^{6}$ o las variantes camín/camiño. Esto resulta curioso, pues Carvalho Calero conoce otros trabajos de Aníbal Otero: de hecho, incluye alguno en los repasos bibliográficos de las diferentes ediciones de su Gramática (aunque no los de léxico, que acaba por retirar de la edición de 1979). Cabe preguntarse, pues, por qué no aparece el ALPI 1962 (e, incluso, por qué retiró los trabajos de léxico de Otero) ni en su Gramática ni (lo que es más intrigante) en sus artículos sobre los dialectos del gallego.

Del mismo modo, en 1967, el académico de la RAG Leandro Carré Alvarellos publica una gramática gallega en la que se ignoran los datos del ALPI 1962 (y los de Zamora Vicente, por cierto) en su descripción de la variación dialectal gallega (Carré Alvarellos 1967: 194-206).

Llama, pues, la atención la escasa presencia del ALPI 1962 en la bibliografía sobre dialectología gallega inmediatamente posterior a su publicación. Y esto es extraño, puesto que al principio de los 60 del siglo pasado era general la queja acerca de la falta de información sobre el gallego dialectal (Piñeiro 2009, Freixeiro Mato 1994).

La primera mención académica de los datos gallegos del ALPI 1962 parece deberse a Manuel Alvar $(1973,1974)$, quien los analizó y llegó a dibujar 7 interesantísimos mapas (recogidos en Alvar 1973). El trabajo de Alvar (1974) aparece en el primer volumen de la revista Verba, fundada por Constantino García, catedrático de Filología Románica de la USC y primer director del Instituto da Lingua Galega (ILG). Curiosamente, Carvalho ignora los útiles mapas publicados por Alvar (1973), que bien habría podido aprovechar para ilustrar sus propuestas dialectales de 1978 y 1979.

\footnotetext{
${ }^{6}$ Por consonantes fricativas coronales entiendo los segmentos fricativos realizados aproximando cualquier parte de la corona de la lengua (ápice, subápice o lámina) a la zona dental, alveolar y postalveolar (Ladefoged \& Maddieson 1996: 19-31).
} 
Años después en Tréveris, Constantino García (1982), al describir las actividades del ILG, menciona el atraso de la lingüística gallega hasta los años 60 del siglo XX y se refiere al Atlas Lingüístico Galego ( $A L G a$ ) como "un dos proxectos mais ambiciosos" del ILG; hay también en su texto una pequeña mención al ALPI 1962: "no que se refire á xeografía lingüística apenas contabamos con mais material có publicado no ALPI, o que é evidentemente insuficiente" (García 1982: 32).

Por su parte, en el mismo encuentro científico, Antón Santamarina sostiene que

Os traballos de dialectoloxía galega empézanse propiamente coas investigacións do ALPI, con inquéritos sistemáticos desde 1931 a 1936 e desde 1947 a 1954. Realmente o ALPI non chegou a proporcionar ós investigadores senón o vol. I, publicado en 1962, cun total de 75 cartas de carácter fonético; eso explica a cantidade escasa de traballos que del derivaron. (Santamarina 1982: 164)

De hecho, trabajos pioneros en la descripción de hablas locales (monografías dialectales) como Couceiro (1976), Porto Dapena (1977) o Taboada (1979) no incluyen referencias al ALPI 1962. Con la excepción del trabajo de Santamarina (1982), las primeras introducciones generales a los dialectos gallegos generadas a partir de datos del ALGa, coma la de Álvarez, Fernández Rei \& Xove (1980), tampoco se refieren en ningún momento al ALPI 1962. Del mismo modo, Fernández Rei (1990) prácticamente solo lo menciona en los pies de los mapas referidos a las fronteras del gallego con el asturleonés y el portugués. La única descripción del ALPI 1962 aparece entre paréntesis en la bibliografía: “(75 mapas. De Galicia hai 52 puntos: 15 na Coruña, 13 en Lugo, 11 en Ourense e 13 en Pontevedra. Practicamente todo o material foi recollido por Aníbal Otero entre 1931 e 1936 e entre 1947 e 1954)” (Fernández Rei 1990: 238); no menciona el número de puntos de habla gallega de Asturias (5), León (2) y Zamora (2); como además existen un punto 112 Lavacolla y otro 112bis Muros, realmente las localidades gallegas son 53, no 52. De hecho, en sus exhaustivas exposiciones sobre la gheada y las consonantes fricativas coronales gallegas, tampoco aparecen los mapas 
originales que ALPI 1962 dedica a estos fenómenos, ni los mapas reelaborados por Alvar (1973). ${ }^{7}$

En sus repasos históricos de la lingüística y la dialectología gallegas, Regueira $(1996,2008)$ tampoco incluye el ALPI 1962. Por su parte, en su exhaustiva historia de la dialectología gallega, Sánchez Rei (2011: 315-316) afirma que "o comezo dos traballos do ALPI durante os anos 1931-1936 e, após a sublevación fascista e posterior guerra, retomados nos anos 1947-1954 na época inicial do franquismo, sitúa a Galiza na cartografía lingüística, mais dun xeito impreciso". Sin embargo, no puedo concordar con la expresión dun xeito impreciso, pues en el $A L P I$, el gallego cuenta con 62 puntos (53 en Galicia y los 9 restantes de habla gallega de Asturias, León y Zamora), mientras que el resto de las hablas gallegoportuguesas cuenta con $94:{ }^{8}$ de este modo, son gallegos casi el $40 \%$ de los puntos gallegoportugueses. Esta mayor densidad de localidades investigadas se debe a que:

En la determinación de los puntos a estudiar, fueron tenidas en cuenta todas las noticias sobre los hechos de que se disponía con anterioridad, para averiguar qué localidades eran más interesantes, y al mismo tiempo, se atendió cumplidamente tanto a los determinantes geográficos, como a los hechos históricos. Ocurre por ello, que la red de las localidades estudiadas es poco simétrica, pues que es más tupida en las zonas de intensa diversidad dialectal, como Asturias, que en regiones de mayor nivelación lingüística, como el centro de Castilla. (ALPI 1962)

En el caso de que dun xeito impreciso se refiera a que, efectivamente, en el ALPI 1962 solo había 70 mapas, es preciso destacar que este hecho afectaba no solo al gallego, sino a todos los dialectos iberorrománicos allí recogidos.

Es claro que el olvido de estos escasos 70 mapas en los trabajos que iniciaron la tradición lingüística gallega contemporánea supuso su preterición en los trabajos de investigación posteriores. Por ejemplo, hasta muy recientemente, el autor de estas

\footnotetext{
${ }^{7}$ Con todo, Fernández Rei (2002), por ejemplo, utiliza el ALPI 1962.

891 puntos en Portugal (pues se deben excluir 221 Rio de Onor y 225 Vimioso, de habla leonesa) y 3 puntos en Cáceres ( 360 Valverde del Fresno, 362 As Ellas y 365 Ferreira), lo que hace un total de 94 puntos gallegoportugueses de habla no gallega. Según Xulio Sousa, las localidades de habla gallega analizadas "fan do territorio galego unha das áreas mellor exploradas no proxecto, tanto tendo en conta a poboación que Galicia tiña daquela coma a superficie" (2008: 304; véase también Sousa en prensa 2).
} 
Dialectologia. Special issue, IX (2021), 39-67.

ISSN: 2013-2247

líneas casi nunca ha citado el ALPI 1962 en sus trabajos, incluso cuando tocaba temas para los que estos datos eran valiosos y relevantes.

\section{Razones del olvido}

Las razones básicas de este olvido pueden ser varias y no queda más remedio que ser especulativo. De este modo, se podría pensar que, al tener su origen en un proyecto madrileño, el ALPI 1962 no se consideraba dialectología gallega. Con todo, cabe empezar diciendo que, como no podía ser de otro modo, los lingüistas gallegos consideran lingüística gallega la que versa sobre el gallego, no la que realizan o dirigen gallegos (de ahí que entre los trabajos iniciadores de la dialectología gallega aparezcan Fritz Krüger, Hans-Karl Schneider, Walter Ebeling, Zamora Vicente o Dámaso Alonso); de hecho, como veíamos arriba, Santamarina dice explícitamente que "os traballos de dialectoloxía galega empézanse propiamente coas investigacións do ALPI". Por lo tanto, el ALPI 1962 es considerado dialectología gallega. Por lo tanto, es posible que la explicación de su olvido provenga de un cúmulo de motivos que conviene separar, pero que en su enunciación no sobrepasan el valor de meras hipótesis.

En el caso concreto de la Gramática de Carvalho Calero, es posible que su desinterés se debiese a que su prioridad fue siempre crear una variedad normativa para la lengua gallega escrita; para él, esta variedad debía basarse, sobre todo, en la lengua literaria y no en los dialectos, de los que tenía una visión negativa (Monteagudo 2020); con aquella finalidad y esta visión, un conocimiento más profundo de la realidad dialectal no tendría por qué verse como apremiante. A pesar de ello, los datos del ALPI 1962 deberían haberle sido ya imprescindibles para sus trabajos sobre dialectología (1969, 1978); resulta difícil saber por qué los ignoró en trabajos de estas características.

Para otros autores, quizás no tan preocupados por la creación de una variedad estándar a partir de la lengua literaria y más pendientes de las variedades populares, las razones deben de haber sido otras. De un lado está la exigua cantidad de mapas, 70, como ya señaló Santamarina (1982). A esto hay que añadir que varios mapas 
ilustran la extensión de los mismos fenómenos, por lo que pueden resultar reiterativos (por lo menos, si los contemplamos desde una perspectiva gallega). Colón \& Lüdtke (1965: 335) señalan que, de esos 70 mapas, solo 36 "ofrecen base adecuada para un estudio fonético comparativo que abrace el conjunto de los tres dominios hispanos", pues en los otros mapas, o bien faltan muchas respuestas (como en el mapa 60 cuévano) o las respuestas no permiten comparaciones fonéticas, ya que las respuestas provienen de étimos distintos (en el mapa 11 aguijón se recogen aguillón, ferrete, rejón...). ${ }^{9}$

Más aún, algunos de los fenómenos de variación dialectal fonética que se pueden analizar en este primer volumen pueden tener interés desde una perspectiva comparativa románica, pero no todos son atractivos desde una perspectiva iberorrománica en general $y$, mucho menos, desde una perspectiva occidental en particular, pues no reflejan bien la diversidad lingüística interna de la península. Así pues, faltan mapas que reflejen fenómenos interesantes como el tratamiento de $-\mathrm{N}-\mathrm{y}$ -L- intervocálicos (gran/grao, grano; verán/verao, verano; muíño, molino) o el de PL- o CL- iniciales (chorar, llorar; chave, llave...), a pesar de que había datos en los cuestionarios. Por su parte, mapas como andar (mapa 15), araña (mapa 16) o cántaro (mapa 34), poca información fonética relevante dan para el gallego (aunque cántaro muestre muchas localidades portuguesas con desaparición de la vocal postónica medial: cantro). Este problema parece deberse a que los lemas seleccionados "van ordenados por orden alfabético, según la palabra castellana" (Colón \& Lüdtke 1965: 332), de modo que no parece que se hayan seleccionado de acuerdo con algún tipo de lógica basada en hechos lingüísticos (vocalismo tónico o átono, consonantismo inicial o final, por ejemplo, como aparecen en las gramáticas históricas; cfr. ALGa III).

Los investigadores gallegos interesados en el cambio lingüístico interno (no debido al contacto) pudieron sentirse sorprendidos al comprobar que algunos de los mapas seleccionados contienen como respuestas en el territorio gallego abundantes castellanismos que eliminaron formas patrimoniales gallegas. La presencia de estos préstamos, aunque es muy informativa sobre la historia de la lengua y tiene

\footnotetext{
${ }^{9}$ A esto se unen decisiones extrañas: el mapa 19 clavo recoge respuestas a la noción castellana de clavo ('pieza metálica para clavar') y a la portuguesa de cravo 'clavel', de modo que se pregunta por la forma y no por el significado.
} 
incuestionable valor, impide comprobar el tratamiento patrimonial que se les dio a las formas gallegas suplantadas por medio del estudio de los cambios internos: es el caso de castillo (mapa 37), cuya presencia impide comprobar si la forma patrimonial castelo se pronunciaría hoy con [e] o con [ع] tónicos; de abuelo (mapa 3), en que la abundante presencia del castellanismo abuelo suplantando las formas gallegas avó, avolo y volo, impide comprobar el tratamiento de la vocal tónica, la átona inicial o la -Lintervocálica; o de ayunar (mapa 21), en que el castellanismo no permite, por ejemplo, comprobar el tratamiento patrimonial gallego a la yod inicial (comparar con el portugués jejuar), aunque formas como aunar muestran cambios gallegos introducidos en un castellanismo.

Por otra parte, Zamora Vicente (1986 [1951], 1986 [1952], 1986 [1953], 1986 [1963]) había conseguido trazar, con anterioridad y con independencia de los datos del ALPI 1962, mapas certeros sobre la difusión de cuatro fenómenos estrella de la dialectología gallega: el seseo, la gheada, los resultados de -ĂNUM y distribución de oi/ui en palabras como moito 'mucho'. ${ }^{10}$ Este hecho pudo desanimar a los investigadores gallegos a tener que bucear en la rica información extraer la información más rica

Otro problema ya bien conocido de los datos del ALPI 1962 tiene que ver con las propias transcripciones fonéticas. Es bien sabido que el sistema de transcripción fonética del ALPI 1962 es extremamente complicado y detallista. Por ejemplo, en la rama de vocales velares redondeadas tónicas distingue doce grados de abertura: en el tipo de sonidos transcritos con [u] aparecen las categorías "de abertura mínima" (1), "abierta" (2) y "dobleabierta" (3); en el tipo de sonidos transcritos con [o] aparecen las categorías "cerrada" (4), "cerrada con tendencia a media" (5), "media con tendencia a cerrada" (6), "media" (7), "media con tendencia a abierta" (8), "abierta con tendencia a media" (9), “abierta" (10), intermedia entre la abierta y la dobleabierta (11) y

\footnotetext{
${ }^{10}$ Zamora Vicente indica que obtuvo sus datos, bien "visitando numerosos lugares (sobre todo en las provincias de Coruña y Pontevedra) en viajes realizados en los años 1942-1945", bien "por medio de informes y datos de personas procedentes de esos lugares, sobre todo a través de alumnos de la Universidad compostelana". Lo cierto es que su red es más densa que la del ALPI y, con todo, humildemente Zamora Vicente teme que "el resultado a que yo llego será rectificado en ulteriores trabajos o a la aparición del ansiado Atlas Lingüístico de España" (1986 [1952]: 13-14, passim; véase también Fernández Rei 2017). Los datos de Zamora Vicente fueron usados por Cintra (1971) para separar los dialectos gallegos occidentales de los orientales.
} 
"dobleabierta" (12). Los propios editores del volumen señalan que una "de las características del ALPI es el rigor y detalle de su sistema de transcripción fonética, más minucioso que el de los demás Atlas románicos". Se pretendía transcribir los sonidos del lenguaje desde una perspectiva absoluta comparativa universal, procurando escapar del sesgo con que la fonología de cada variedad pudiese condicionar la descripción estrictamente fonética:

Conviene recordar que nuestro alfabeto fonético radica sobre articulaciones tipo claramente identificadas en el uso internacional, y no sobre las bases de los sistemas fonológicos de las lenguas hispánicas. Así, por ejemplo, el signo ę representa el sonido de la è abierta francesa de fer, italiana de ferro, y el signo é la francesa beauté, aunque ambas articulaciones sean insólitas en español. En el dominio catalán, los fonemas de é y ó cerradas de esa lengua, son articulaciones generalmente con en é y ó simplemente medias, y en algunas regiones con $e_{\text {y }} \underline{Q}$ medias con tendencia a abierta, muy lejanas de las e y $o$ cerradas del francés, alemán, etc.; en dichas regiones, los fonemas catalanes è y ò abiertas, suelen pronunciarse $e$ y $q$ dobleabiertas. (Sanchis Guarner et al. 1961: 118)

El propósito de esta minuciosidad era detectar cambios en marcha o rasgos fonéticos (no necesariamente distintivos) que covariasen con rasgos extralingüísticos. Sin embargo, todo parece indicar que, como señalan Colón \& Lüdtke (1965: 327), los editores mantienen una "creencia excesiva en la objetividad y universalidad de los símbolos fonéticos"; de hecho, Colón \& Lüdtke (1965: 330) llegan a preguntarse con razón si "es capaz un investigador de observar una matización tan extremada a lo largo de toda la encuesta en su territorio, sin riesgo de trocar matices afines" y si, por consiguiente, castellano y catalán presentan efectivamente diferencias lingüísticamente significativas en la articulación de las consonantes africadas de la palabra coche/cotxe (p. 331). En el mismo sentido se pronuncia Lope Blanch (1963: 248-249); véase también Pérez Pascual (2016: 155-157) para las controversias entre los entrevistadores.

Es posible que este intento de precisión esté fundamentado teóricamente en la visión neogramática del cambio fonético como una acumulación de pequeñas diferencias articulatorias paulatinas e inconscientes que se van sumando poco a poco 
durante el paso gradual de un segmento fonético a otro (véase McMahon 1994: 49; Trask 1996: 294-296; Hock 1991: 630-636); de ahí que se proponga la existencia de una "consonante bilabio-labiodental fricativa sonora, intermedia entre $v$ y $b$ ", un segmento que, a mi juicio, causa perplejidad; supongo que este punto de articulación doble se considera un paso necesario intermedio entre una fricativa bilabial y una labiodental (véase Ladefoged \& Maddieson 1996: 139-140). En todo caso, al final Colón \& Lüdtke (1965) concluyen que:

Del estudio de la transcripción deducimos que los materiales de la parte fonética del ALPI se deben utilizar con cierta cautela, a pesar de que los investigadores, por su previa familiaridad con el idioma estudiado, no habrán caído en errores importantes (como puede suceder cuando un investigador desconoce el sistema fonológico del habla en cuestión). (Colón \& Lüdtke 1965: 331).

Efectivamente, es preciso observar los datos fonéticos del ALPI 1962 con cautela, aunque esto no los invalida radicalmente. Por ejemplo, no deja de causar perplejidad que en el mapa 11 aguijón aparezcan no pocos puntos de habla gallega con una vocal media tónica con tendencia a abierta en aguillón (cuando, salvo en el Bajo Miño y en Asturias, esta terminación tiene mayoritariamente una vocal media cerrada); por su parte, onte, en el mapa 20 ayer, tiene una vocal tónica abierta con tendencia a media en varios puntos; o que cabeza (mapa 30) pueda aparecer con una vocal media tónica con tendencia a abierta, mientras que diez (mapa 70) tenga en puntos similares abierta tónica con tendencia a media, lo que reduce dos vocales bien distintas a una diferencia articulatoria mínima; lo mismo con los abundantes cazador (mapa 39) con vocal media tónica con tendencia a abierta. Quizás todo este problema esté relacionado con distinguir ocho grados de abertura solo en las vocales que hoy llamamos medias. Por otra parte, el mapa 22 azada recoge respuestas como legón/ligón, que en varios puntos presenta un diptongo tónico decreciente [õ w] con la semivocal relajada; este diptongo no ha sido descrito en otras ocasiones en territorio gallego. Finalmente, llama la atención que, con tanta precisión fonética, los investigadores del ALPI no oyesen y 
categorizasen al transcribir el habla real los segmentos $[\underset{T}{\beta}, \underset{T}{\sigma}, \underset{T}{\gamma}]$ que hoy llamamos aproximantes, consonantes sonoras continuas sin ruido de fricción. ${ }^{11}$

Como bien señala Manuel Alvar (1973: 195-196) cuando reflexiona sobre el grado de precisión en las transcripciones fonéticas:

No se olvide nunca que el sonido es el elemento primario de nuestro conocimiento, sin su recto valor fallarán todas las especulaciones posteriores; pero tengamos presente que tanto lo adultera la caracterización incompleta de sus rasgos distintivos como la artificiosa atomización de los hechos. ${ }^{12}$ (Alvar 1973: 195-196)

En un sentido similar parece manifestarse Lass (1997: 221-225) cuando reflexiona sobre cuánto es necesario segmentar el espacio articulatorio (o acústico) para dar cuenta de los cambios fonéticos, o sobre si existen cambios bruscos, o cambios graduales:

There is of course one serious methodological issue: the other side of the coin is the finesse of a given phonetic ear. One phonetician who could hear more gradations the another might legitimately operate with a finer scale of primitives, and this would produce anarchy rather than science. (Lass 1997: 222)

De hecho, es notorio que existían desacuerdos entre los investigadores a la hora de transcribir los datos (Pérez Pascual 2016: 155-157). Es posible que este complejo (y a veces sorprendente) sistema de descripción fonética del ALPI 1962 bien desesperase a más de un investigador, bien lo hiciese desconfiar. En todo caso, como ya dije arriba,

\footnotetext{
${ }^{11}$ Nótese que para el punto de articulación dental detectan una "fricativa sonora relajada, con menor aproximación del ápice de la lengua a los incisivos que en $d^{\prime \prime}$; pero no aclaran si, frente a la fricativa no relajada, carece o no de ruido de fricción, que es por lo que se puede identificar este segmento, hoy presente en cualquier manual de fonética española. No hay tal grado de constricción para el punto de articulación velar ni para el bilabial.

${ }^{12}$ Esto no significa que efectivamente no se puedan percibir pequeñas diferencias en las realizaciones de las vocales: el sistema de transcripción del $A L G a$ distingue seis vocales medias tónicas, [o], [o], [ơ], [ग̣], [0] y [?] (ALGa III: 19). Muchos trabajos de sociolingüística analizan vocales detectadas en estos contínuums (Labov 1983 [1972]: 45). La cuestión es si es posible ser siempre coherente, acertar siempre en una discriminación tan fina, entre tal masa de datos y con encuestadores diferentes, y si la ganancia en el detalle compensa el esfuerzo; o también si el investigador que consulte el atlas le va a sacar provecho a 12 grados de abertura sólo en las vocales velares tónicas (a los que hay que sumar diferentes grados de centralización, nasalidad, sonoridad, etc.).
} 
que se deba usar el ALPI 1962 con cautela no significa que no se use o que no sea informativo, y no explica su ausencia en las bibliografías de dialectología gallega, pues da información abundante para fenómenos con diferencias más gruesas y perceptibles (seseo, gheada, sonoridad de las fricativas coronales, etc.).

Quizás otro factor que contribuyese al olvido de los 70 mapas del ALPI 1962 pudo ser la opinión dominante en la época de que el momento de los atlas de grandes dominios había pasado a la historia. Para Alvar (1973: 199), era hora "de buscar la riqueza local, difícilmente compilada en los Atlas de grandes dominios" (1973: 193). Con todo, como he mostrado más arriba, en los cuestionarios del ALPI había algunas preguntas que permitirían estudiar la distribución de variantes lingüísticas en el dominio gallego que con el tiempo resultaron tópicos típicos de nuestra dialectología (además de los comentados sobre el seseo, la gheada, la variación gran/grao, con los datos del ALPI se podrían hacer, por ejemplo, mapas sobre el plural de los nombres agudos acabados en nasal: mapa 366 se castigó a los ladrones, mapa 579e chicharrones). Por otra parte, la escasez de información sobre el gallego hablado que todos reconocían a la altura de 1962 transformaba este volumen en un tesoro en sí mismo: basta con ver las descripciones de la realidad dialectal gallega elaboradas antes de la publicación del ALPI 1962 (un buen resumen en Sánchez Rei 2011).

En fin, y ya con posterioridad a los años 70, otro motivo para el olvido del $A L P I$ 1962 (y del ALPI en general) quizás fuese la ilusión y el trabajo puestos en esa inmensa base de datos que acabaría siendo el $A L G a$.

\section{La información dialectal que el ALPI 1962 contiene}

Con todos los posibles defectos que el proyecto del $A L P I$ pudiese tener, y con toda la precaución con que hubiese que contemplar sus datos, es un hecho que contenía información geolectal valiosa, fiable y abundante para la morfología y, sobre todo, la fonética gallegas. Evidentemente, en mucha menor medida, ofrecía algo de información sobre la variación léxica que aquí tengo que ignorar; en este sentido, es preciso indicar que la información sobre la variación léxica incluye no solo la 
distribución geográfica de las variantes gallegas, sino también la distribución geográfica de los castellanismos, que merece por si sola un acercamiento detenido. Veamos algunos ejemplos, centrándonos solo en los datos correspondientes a las hablas gallegas. Daré siempre que pueda mapas similares del $A L G a$ o remitiré al trabajo de Fernández Rei (1990).

\subsection{En la morfología}

El ALPI 1962 nos informa sobre variaciones geolingüísticas en la vocal temática seleccionada para crear el tema de seis substantivos:

Mapa 14, aire. Se observa la presencia de una vocal temática /o/ en la variante airo del sustantivo en el suroeste de Galicia, frente a la mayoritaria /e/ de aire. El mapa también muestra que la raíz usada en gallego es air-, frente a la portuguesa ar- (en Portugal se recoge aire en Rio de Onor, punto 221, y Duas Igrejas, punto 225). Cfr. ALGa II, mapa 22 aire.

Mapa 17, árboles. Ofrece alternancias similares a la anterior en la vocal temática del plural del sustantivo árbore: árboles, árbolos (Corcubión, punto 108), árboros, arbos (Fornelos de Montes, punto 135), árboes (Vilamarín, punto 142). Cfr. ALGa VI, mapa 178 árbores.

Mapa 45, chinche. Además de recoger información léxica (presebello, chíncharra, rubico), muestra la existencia aislada de formas con vocal temática /e/ (chinche) y la presencia mayoritaria de formas con vocal temática /a/ (chincha); un caso de /o/ (chincho). Desgraciadamente, no informa del género. Cfr. ALGa II, mapa 24 chinche.

Mapa 51, coz. Se percibe la presencia en el suroeste (en Nigrán, punto 136, y Fornelos de Montes, punto 135) de formas couzo, con vocal temática /o/, con continuidad en el noroeste portugués (véase Figura 1); existencia de formas atemáticas couz, sobre todo en Asturias, con continuidad en el leonés. Cfr. ALGa III, mapa 350 couce. 


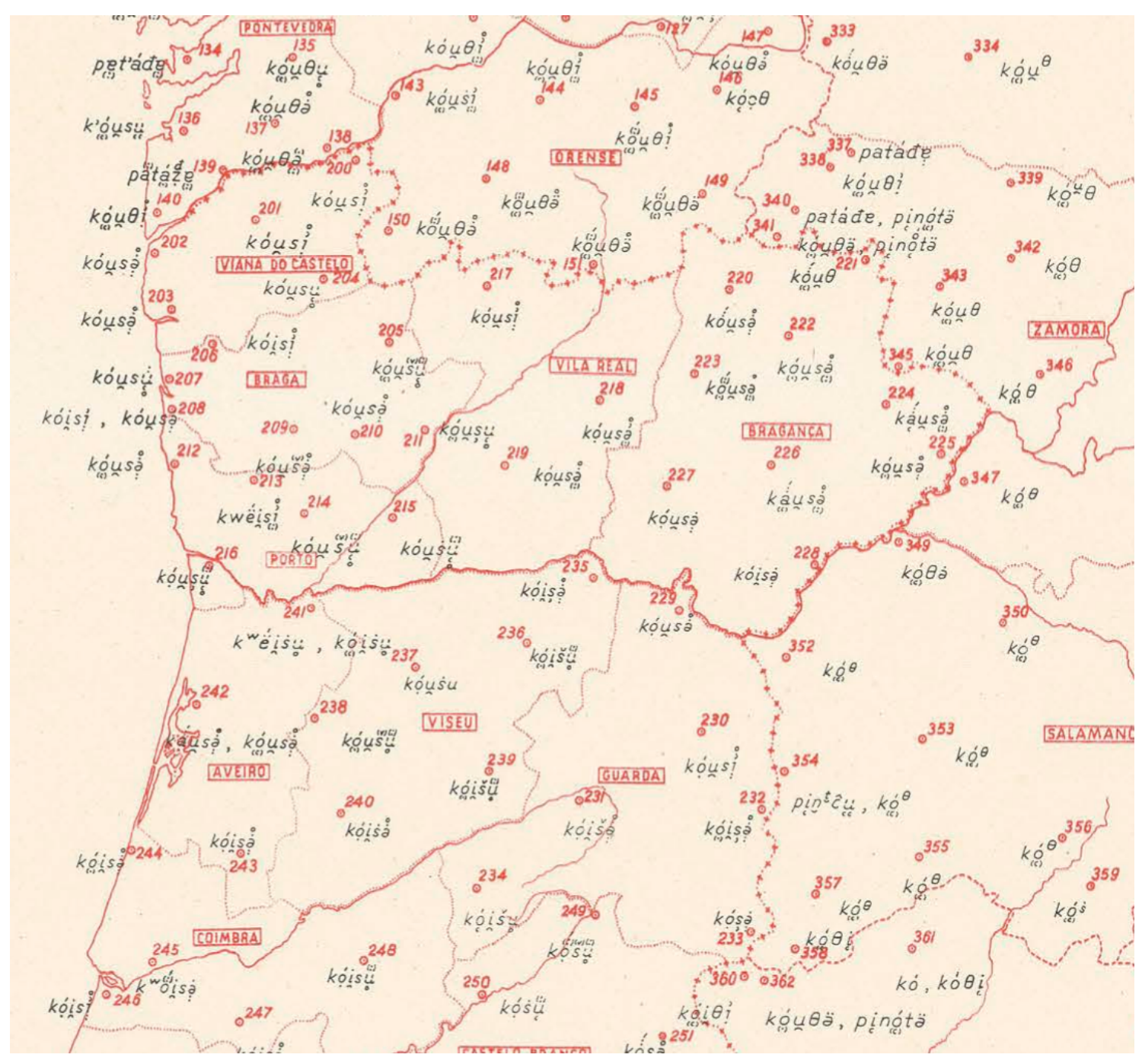

Figura 1. Formas tipo couzo/couço 'coz' en gallego y portugués. Fuente: Mapa 51 coz de ALPI 1962.

Mapa 53, crin. Vemos la presencia mayoritaria de formas con vocal temática /a/ (clina), algunas con vocal temática /e/ (cline) y de formas atemáticas (clin) en el occidente. Las formas con vocal temática /a/ agrupan gallego, portugués y asturleonés frente al castellano. Cfr. ALGa II, mapa 52 crin.

Mapa 75, eje. Muestra en casi todo el occidente (excepto el occidente coruñés) la existencia de formas con vocal temática /o/, eixo. Es también interesante indicar que las formas con vocal temática /e/ aparecen en la mitad oriental de Galicia, el noreste portugués (Vila Real, Bragança) y todo el leonés y castellano (véase Figura 2). Cfr. ALGa II, mapa 21 eixe. 


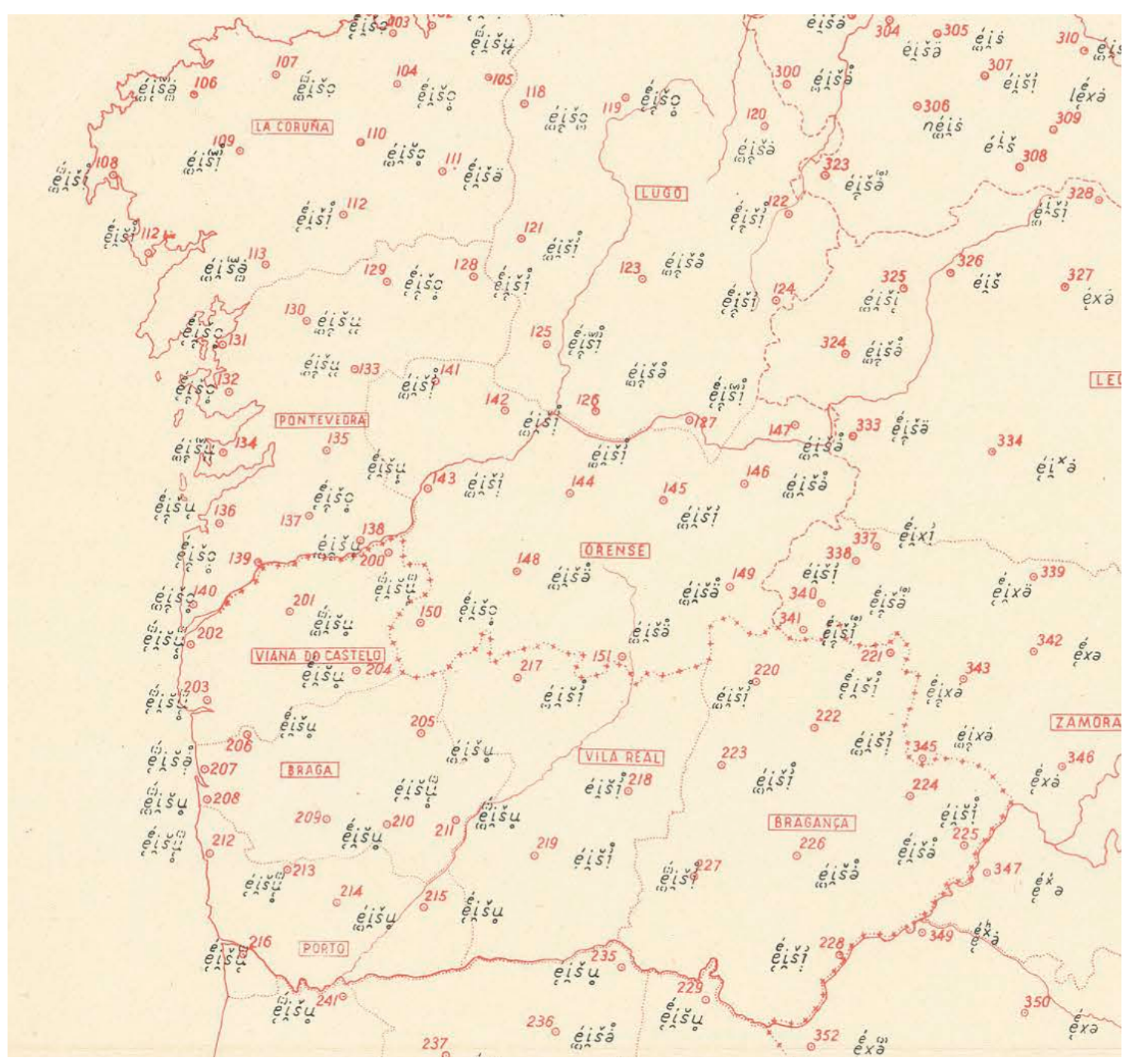

Figura 2. Distribución de las formas tipo eixe, con vocal temática - $e$, en gallego, asturleonés y portugués. Fuente: Mapa 75 eje de ALPI 1962.

Asimismo, contiene información sobre la conjugación a que pertenecen dos verbos:

Mapa 62, decirlo. Presenta algunos casos de adscripción a la segunda conjugación del lexema DICIR 'decir' en el suroeste de Ourense; resulta curioso que no se documentase este fenómeno en el occidente de Coruña, donde todavía hoy sigue vivo. Cfr. ALGa I, mapa 242 dicir; Fernández Rei (1990: 93-95).

Mapa 31, caer. Permite establecer la isoglosa de la atribución a la segunda conjugación (caer) o a la tercera (caír) del lexema verbal CAER (esta última con continuidad en el portugués); muestra la existencia de una forma contracta (quer ['ker]) en Asturias (Boal, punto 303) y de formas de la segunda conjugación con 
semivocal antihiática (caier [ka'jer]), que también aparecen en asturleonés. Cfr. ALGa I, mapa 160 caer; Fernández Rei (1990: 93).

Informa también sobre la realización de dos adverbios.

Mapa 20, onte. Comprobamos la presencia compacta de una forma ontes, con /s/ analógica de la de otros adverbios (como antes, despois, antiguo cras 'mañana') en todo el norte de Galicia; y la existencia en Asturias (Freal, punto 302) de una forma [a'nojte], con una nasal velar epentética similar a la de engalar o engadir. Como curiosidad, se puede señalar que en los distritos de Viana do Castelo y Braga aparecen formas tipo onte, con vocal tónica nasal media abierta y monoptongo final oral: [ग̃nti̇]. Cfr. ALGa II, mapa 25 onte; Fernández Rei (1990: 103).

Mapa 73, ¿donde? Permite observar la altura de la vocal tónica, así como diversos modos de contracción del adverbio con las preposiciones que los anteceden: unde en el gallego de Asturias, o vocales tónicas medias abiertas fruto de la contracción con $a$ o con la preposición pra por todo el territorio.

En definitiva, el ALPI 1962 contenía varios mapas sobre el vínculo entre género del substantivo y la vocal temática, que permiten reflexionar sobre el cambio analógico. También contenía información sobre la conjugación de los verbos dicir/decer y caer/caír.

\subsection{En la fonética}

Dejando claro que las transcripciones fonéticas del ALPI 1962 deben ser contempladas con cautela, será fácil mostrar que no era despreciable la cantidad de información sobre los fenómenos de variación geolingüística fonética gallega que ofrecían estos 70 mapas.

En términos generales, se aprecia la realización reducida, centralizada y ensordecida de las vocales átonas finales, fenómeno que fue largamente ignorado en las descripciones fonéticas generalistas del gallego.

Seguidamente, ofrezco las variables dialectales fonéticas cartografiadas en el ALPI 1962 con los nombres que la tradición dialectológica gallega les ha otorgado 
(cuando los tengan, claro está); el origen de la mayoría de estos fenómenos de variación está explicado por Mariño Paz (2017).

Alteración del timbre etimológico de las vocales medias tónicas (Fernández Rei

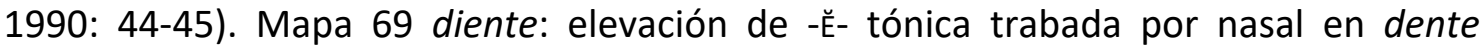
(d['ẽ]nte occidental vs. $d[$ ['z̃]nte oriental). Cfr. ALGa III, mapa 63 dente.

Evolución de los grupos latinos -ŬLT-, -ŬCT-, etc. (Fernández Rei (1990: 48-51). Mapa 58 cuchillo: tratamiento del grupo latino -ŬLT- pretónico (coitelo, cuitelo, cutielo, cutelo). Mapa 59 cuero: tratamiento del grupo latino -ŏRI- tónico (coiro, cuiro, couro). Cfr. ALGa III, mapas 19 coitelo y 40 coiro.

Mapa 63 dedo: presencia de formas tipo dido en todo el gallego de Asturias y en puntos orientales de Lugo (Devesa, punto 117, y Neiro, punto 120); Álvarez (1988: 150) y Mariño Paz (2017: 177) consideran esta forma un préstamo asturiano. Cr. ALGa III, mapa 114 dedo.

Mapas 43 cereza y 64 dereita: tratamiento de la vocal media anterior pretónica, cereixa, cireixa, zreixa; dereita, direita, dreita. ${ }^{13} \mathrm{Cfr}$. ALGa VI, mapa 230 cereixa.

Mapas 55 cuadrado, 61 cuñados y 65 desbocado: mantenimiento generalizado de /d/ intervocálica en la terminación -ado; en estos mapas no se localiza ningún punto en Galicia en que haya caído la /d/ intervocálica; si existen formas tipo cuñaus en el gallego de Asturias. Es interesante también notar que en varios puntos (en estos mapas y otros con /d/ intervocálica) se recogen abundantes realizaciones aparentemente fricativas (descritas como consonante dento-interdental fricativa sonora, intermedia entre $₫ \mathrm{y}$ z) para /d/ precedida de vocal, algo que se puede apreciar a veces en algunos hablantes de gallego hoy en día.

Resultados de /ts/ prenuclear medieval; seseo (Fernández Rei 1990: 54-56 y 189200). Mapas 9 acero, 27 brazo, 30 cabeza, 39 cazador, 40 cazuela, 41 cejas, 43 cereixa, 46 cincha, 47 cinco y 74 dulce: variación de dental no sibilante [ $\theta]$ frente a diferentes tipos de sibilantes anteriores sordas (apicales o laminales, dentales o alveolares) en el occidente gallego como realización de una consonante fricativa coronal; por lo tanto, ilustra la presencia/ausencia del seseo; se debe notar que esta fricativa dental $[\theta]$ no

\footnotetext{
${ }^{13}$ El mapa 43 cereza permite ver la extensión del grupo $[\theta r]$ en respuestas como zreixa.
} 
sibilante es común en Entrimo (punto 150) ${ }^{14}$ y en Hermisende (punto 341); en Arnoia (punto 143) aparece constantemente una fricativa sibilante apicoalveolar en estas palabras. ${ }^{15}$ Cfr. González González (1991); ALGa III, mapa 412 brazo; ALGa II, mapa 285 cinco.

Resultados de /dz/ prenuclear medieval; seseo y sibilantes sonoras (Fernández Rei 1990: 54-56 y 189-200). Mapas 49 cociña, 62 decirlo y 71 doce: además de los tipos de seseo en los puntos donde solo hay fricativas sibilantes sordas, muestra la existencia de una sibilante interdental sonora en cociña o en doce en Entrimo, y en doce en Hermisende; para decirlo aparece una sibilante laminoalveolar sonora en Entrimo y una apicoalveolar sonora en Hermisende; muestra seseo sordo apical también en Arnoia. Cfr. González González (1991); ALGa III, mapa 171 dicir.

Resultados de /žl prenuclear medieval; sibilantes sonoras (Fernández Rei 1990: 54-56 y 189-200). Mapas 18 asa y 38 causa: presencia de fricativas alveolares sonoras en Entrimo y en Hermisende; del mismo modo, puntos de articulación apicoalveolares sordas generalizadas en el resto del territorio, con laminoalveolares o apicodentales en el occidente. Cfr. ALGa III, mapa 196 casa.

Resultados de /ș/ prenuclear medieval (Fernández Rei 1990: 54-56 y 189-200). Mapas 13 ahogarse y 41 cejas (en sibilante prenuclear de las respuestas tipo sobrecellas): sibilantes apicales (en general) o laminales (en el occidente) sordas como realización de la fricativa alveolar; aparición de alguna apicopostalveolar en el centro de Galicia (puntos 105, Aranga; 111, Boimorto; 112, Lavacolla); puntos de articulación laminoalvolares o apicodentales con fonación sorda aparecen en el occidente en el mapa 38 causa. Cfr. ALGa II, mapa 282 sete.

Palatalización de /s/ posnuclear (Fernández Rei 1990: 56). Mapa 17 árboles: presencia de fricativas apicoalveolares y alguna ápicopostalveolar en el noroeste de Coruña (puntos $105,111,112$ ) como morfo de plural.

Resultados de /ts/ posnuclear medieval; seseo posnuclear (Fernández Rei 1990: 54-56 y 189-200). Mapas 54 cruz y 70 diez: muestra la existencia de seseo posnuclear,

\footnotetext{
${ }^{14}$ En Entrimo se recoge una sibilante laminodental en cazador.

${ }^{15}$ Zamora Vicente (1986 [1951]: 3) todavía detectaba en sus datos de los años 40 del siglo XX una "laguna seseante" en el habla de los viejos de Arnoia.
} 
ausencia de $[\theta]$ en la coda silábica en el occidente gallego y presencia de una sibilante coronal en su lugar (con abundantes casos de realizaciones apicopostalveolares). Cfr. ALGa III, mapa 174 cruz.

Rotacismo de /s/ posnuclear (Fernández Rei 1990: 57). Mapas 65 desbocado, 66 desnudo y 72 los domingos: ilustran la existencia de rotacismo de /s/ posnuclear, con la realización de /s/ en coda como [r] ante consonantes sonoras; este fenómeno, que aparece en otros dialectos ibéricos sin conexión geográfica con el gallego, es mucho más común en el mapa 72 los domingos que en los otros dos. Cfr. ALGa III, mapa 199 os dedos.

Despalatalización de /J/ prenuclear (Fernández Rei 1990: 56). Mapas 22 azada, 32 caja, 50 coxo y 75 eixe: presencia de apicopostalveolares en el occidente de Coruña (puntos 106, Baio; 109, Santa Comba; 113, Rois). Cfr. ALGa III, mapas 49 caixa, 334 eixe.

Despalatalización de /J/ prenuclear y preservación de /3/ prenuclear medieval (Fernández Rei 1990: 56). Mapa 43 cereza: además de las apicopostalveolares referidas, este mapa muestra la existencia de fricativas sonoras otra vez en Entrimo y en Hermisende. Cfr. ALGa III, mapa 385 queixo; ALGa VI, mapa 230 cereixa.

Distribución ax-/aix- (Fernández Rei 1990: 52). Mapa 32 caja: ausencia de semivocal palatal tras la vocal tónica (caxa, frente a caixa) en las hablas orientales (Asturias y Zamora). Cfr. ALGa III, mapa 49 caixa.

Resultados de las terminaciones latinas -ĨNUM, -ĨNAM; mantenimiento de las vocales nasales medievales (Fernández Rei 1990: 48 y 64). Mapa 33 camino: elisión de la vocal átona final en la terminación latina -ĨNUM en el noreste del dominio, con continuidad en el asturleonés, frente a la caída general de la nasal intervocálica (con generación de una nasal palatal posterior, camiño); presencia de un hiato nasal en Castroquilame (camĩo, punto 333). Mapa 49 cocina: muestra, con pocos datos, el tratamiento de la terminación latina -ĪNAM, generación de nasal palatal (cociña) frente a la presencia de hiatos orales en Asturias (cocía). Cfr. ALGa III, mapas 240 madriña, 241 fariña, 250 veciño.

Yeísmo (Dubert-García 2013). Mapas 1 abeja, 11 aguijón, 29 caballo, 37 castillo, 41 cejas, 44 cerrojo, 57 cuchara, 62 decirlo: ausencia de yeísmo en los años 30, incluso 
en el préstamo castillo; solo aparece yeísmo en Freal (punto 302), gallego más oriental de Asturias. Cfr. ALGa III, mapas 169 agulla, 288 cabalo; ALGa II, mapa 178 cantalo (cantar +0$)$.

Gheada (Fernández Rei 1990: 53-54 y 163-177). Mapas 10 agua, 11 aguijón, 12 agulla, 13 afogarse y 72 los domingos: datos de la extensión de gheada, uno de los fenómenos más conocidos de la dialectología gallega y un auténtico estereotipo, en el sentido laboviano (Labov 1983 [1972]: 387-388); aparecen descritas diferentes realizaciones y cartografiada su extensión geográfica, con gheada en Pedrafita do Cebreiro (punto 124); asimismo, información sobre la realización [k] ante consonante nasal (dominco fronte a domingo). Cfr. ALGa III, mapas 169 agulla, 189 auga, 372 domingo.

Palatalización de -LL- latino (Fernández Rei 1990: 57-58). Mapas 29 caballo, 62 decirlo: palatalización de -LL- en las hablas orientales del gallego de Asturias (caballo, decillo), frente a su simplificación en el resto del gallego y del portugués; en los datos del ALPI 1962 solo aparece yeísmo en Freal. Cfr. ALGa III, mapa 288 cabalo; ALGa II, mapa 178 cantalo (cantar +0$)$.

Mantenimiento de los grupos cua-, gua- (Fernández Rei 1990: 51-52). Mapas 55 cuadrado y 55 cuatro: en las respuestas a cuadrado, llama extraordinariamente la atención la presencia de [w] tras la consonante velar inicial en todo el territorio de habla gallega (cuadrado), pues es común su pérdida en el centro y occidente gallego, como muestran los resultados catro del mapa 55. Cfr. ALGa III, mapas 395 catro.

\section{Conclusiones}

Es claro que en el año 1962 los datos fiables sobre el gallego dialectal eran escasos; los miembros de la RAG y los intelectuales empeñados en crear una variedad normativa de la lengua señalaban este problema constantemente, pues esta era, para muchos de ellos, una de las dificultades que percibían para poder establecer un gallego normativo con fundamento en el habla real. 
Una parte del mundo intelectual galleguista conoció y saludó la publicación de los mapas del ALPI 1962. El mundo académico gallego también conocía esta publicación. Con todo, los datos que la obra ofrecía no fueron explotados ni resultaron atractivos para los investigadores posteriores; de hecho, esta publicación pasó solo a ser citada con prisas en los trabajos introductorios a la dialectología gallega. Un ejemplo claro lo tenemos en la obra de Carvalho Calero, quien al pergeñar su establecimiento de las modalidades dialectales del gallego no tiene jamás en cuenta los mapas que cartografían varias de las variables dialectales que él usa en su propuesta.

En este trabajo he intentado presentar algunas hipótesis que expliquen por qué fue ignorado el ALPI 1962 y mostrar qué datos nos ofrecía. Entre las razones del olvido propongo el interés por crear una variedad estándar a partir de la lengua literaria, la escasez de los mapas publicados y su relativo interés para establecer las variedades internas del gallego, la existencia de los mapas de Zamora Vicente, la cautela con que hay que observar las transcripciones fonéticas (fruto, quizás, de una visión neogramática del cambio lingüístico) y las dificultades que ocasionaba su manejo.

En cuanto a la información recogida en el ALPI 1962, me he centrado solo en los datos morfológicos y los fonéticos, pues son los más ricos y sistematizables. He mostrado como aparecen cartografiados fenómenos interesantes, tales como las reducciones, centralización y ensordecimiento de las vocales átonas finales, fenómenos que fueron descuidados durante mucho tiempo en las descripciones fonéticas gallegas generales; algunos fenómenos que siempre han atraído la atención de los investigadores (gheada, seseo, yeísmo, sibilantes sonoras, conjugación del verbo dicir 'decir'); otros fenómenos que no han sido tan populares, pero que sí resultan interesantes para el estudioso del cambio lingüístico en gallego (las vocales temáticas de algunos sustantivos, la extensión analógica de la -/s/ final en el adverbio onte). Los mapas tienen interés incluso para analizar la distribución geográfica de algunos castellanismos.

Si además abandonamos la perspectiva interna, los datos del ALPI 1962 nos muestran las continuidades de los fenómenos dialectales que aparecen en Galicia, unas veces por Portugal, otras veces por Asturias, León y Zamora, y aún otras por el 
noroeste en general (véanse las Figuras 1 y 2 presentadas arriba); el estudio de estas continuidades permitiría situar a todas estas hablas unas frente a otras y, como consecuencia, enriquecería mucho nuestra visión de la historia del gallego.

La explotación de los datos que nos ofrezca la publicación del ALPI permitirá realizar estudios de cambio en tiempo real (algunos ya se han realizado, por ejemplo, Rodríguez Lorenzo 2011) o estudios areales (como los de Dubert García \& Sousa 2009 o Fernández Ordóñez 2011), que iluminarán la historia del noroeste ibérico.

\section{Referencias bibliográficas}

ALGa I = Instituto da Lingua Galega (1990) Atlas Lingüístico Galego. Vol. I. Morfoloxía verbal, A Coruña: Fundación Pedro Barrié de la Maza.

ALGa II = Instituto da Lingua Galega (1995) Atlas Lingüístico Galego. Vol. II. Morfoloxía non verbal, A Coruña: Fundación Pedro Barrié de la Maza.

ALGa III = Instituto da Lingua Galega (1999) Atlas Lingüístico Galego. Vol. III. Fonética, A Coruña: Fundación Pedro Barrié de la Maza.

ALGa VI = Instituto da Lingua Galega (2015) Atlas Lingüístico Galego. Vol. VI. Léxico. Tierras, plantas e árbores, A Coruña/Santiago de Compostela: Fundación Barrié/Universidade de Santiago de Compostela.

Alonso MonTERO, Xesús (2009) "Para a biografía de Aníbal Otero como lingüista: documentos e datos inéditos ou descoñecidos", Madrygal, 12, 11-24.

ALPI 1962 = Atlas Lingüístico de la Península Ibérica l. Fonética, I, Madrid: Consejo Superior de Investigaciones Científicas.

ALVAR, Manuel (1973) Estructuralismo, geografía lingüística y dialectología, Madrid: Gredos.

ALVAR, Manuel (1974) “Galicia en la cartografía lingüística”, Verba, 1, 54-67.

ÁlVAREZ, Rosario (1988) “Consideracións sobre a metafonía nominal galega”, en Dieter Kremer (ed.), Homenagem a Joseph M. Piel por ocasião do seu 85o aniversário, Tübingen: Max Niemeyer Verlag, 141-157.

Álvarez, Rosario, Francisco Fernández Rel \& Xosé Xove (1980) “Galego", en Gran Enciclopedia Galega, 14, 222-255.

CARBAllo CALERO, Ricardo (1966) Gramática elemental del gallego común, primera edición, Vigo: Galaxia. 
Carballo Calero, Ricardo (1969) "Sobre os dialectos do galego", Grial, 23, 1-15.

Carballo Calero, Ricardo (1978) “Sobre dialectoloxía do galego", Grial, 61, 348-353.

CARballo Calero, Ricardo (1979) Gramática elemental del gallego común, séptima edición, Vigo: Galaxia.

Carballo Calero, Ricardo (1981a) “O idioma no Seminário de Estudos Galegos”, en Problemas da língua galega, Lisboa: Sá da Costa, 111-120.

Carballo Calero, Ricardo (1981b) "Sobre dialectologia do galego", en Problemas da língua galega. Lisboa: Sá da Costa, 121-128.

CARRÉ AlVARELLOS, Leandro (1967) Gramática galega, Coruña: Moret.

CINTRA, Luis F. Lindley (1971) “Nova proposta de classificação dos dialectos galegoportugueses", Boletim de Filologia, 22, 81-116.

COLÓN, Germán \& Helmut LÜDTKE (1965) “[Reseña a] Atlas Lingüístico de la Pensínsula Ibérica, vol. I, Fonética 1. Consejo Superior de Investigaciones Científicas, Madrid, 1962", Vox Romanica, XXIV, 323-335.

CouCEIRo PéreZ, José Luis (1976) El habla de Feás, Santiago de Compostela: Universidade de Santiago de Compostela.

DUbert-García, Francisco \& Xulio Sousa (2009) “Lexical areas in the Iberian Peninsula based on a proposal by Lindley Cintra", VI Congreso de Ia SIDG, celebrado en Maribor, entre el 14 y el 18 de septiembre de 2009.

<https://www.researchgate.net/publication/268198148_LEXICAL_AREAS_IN_THE_IBERI AN_PENINSULA_BASED_ON_A_PROPOSAL_BY_LINDLEY_CINTRA>

Dubert-García, Francisco (2013) "El yeísmo y el fortalecimiento de /j/ en Galicia", en Rosario GonZÁlez \& Isabel Molina MARTos (eds.), Variación yeísta en el mundo hispánico, Frankfurt am Main / Madrid: Vervuert / Iberoamericana, 39-57.

FERNÁNDEZ REI, Francisco (1990) Dialectoloxía da lingua galega, Vigo: Xerais.

FERNÁNDEZ ReI, Francisco (2002) “Do Ortegal ó Douro e de Fisterra ó Navia e ó Padornelo. Notas sobre léxico moderno da Gallaecia e do seu litoral", en Rosario ÁlVAREZ, Francisco DUBERT GARCíA \& Xulio SOUSA (eds.), Dialectoloxía e léxico, Santiago de Compostela: Instituto da Lingua Galega/Consello da Cultura Galega, 147-192.

FERNÁNDEZ REI, Francisco (2017) “Alonso Zamora Vicente, precursor de la dialectología gallega”, Revista de Filología Románica, 34, 19-30. <http://dx.doi.org/10.5209/RFRM.58151>

FERNÁNDEZ-ORDóÑEZ, Inés (2011) La lengua de Castilla y la formación del español. Discurso leído el 13 de febrero de 2011 en su recepción pública en la RAE por la Excma. Sra. D. a Inés Fernández-Ordóñez y contestación del Excmo. Sr. D. José Antonio Pascual, Madrid. 
<http://www.alpi.csic.es/sites/default/files/publicaciones/Discurso_IFO.pdf> FreiXeIRO Mato, Xosé Ramón (1994) Sebastián Martínez Risco na cultura galega, Santiago de Compostela: Laiovento.

GarcíA, Constantino (1982) "Actividades do Instituto da Lingua Galega", en Dieter Kremer \& Ramón LORENZO (eds.) Tradición, actualidade e futuro do galego. Actas do Coloquio de Tréveris, Santiago de Compostela: Xunta de Galicia, 23-34.

GonZÁlez GonZÁlEZ, Manuel (1991) "Subsistema de sibilantes no galego actual", en Dieter Kremer (ed.), Actes du XVIIle Congrès International de Linguistique et de Philologie Romanes (Université de Trèves (Trier) 1986), vol. 3, Tübingen: Max Niemeyer Verlag, 531-548.

Hock, Hans Henrich (1991) Principles of Historical Linguistics, Berlin/NewYork: Mouton de Gruyter.

LABOV, William (1983 [1972]) Sociolinguistic patterns, Philadelphia: University of Pennsylvania Press. Cito por la traducción española Modelos sociolingüísticos, Madrid: Cátedra, 1983.

LAdefoged, Peter \& Ian MAdDieson (1996) The sounds of the World's languages, Oxford: Blackwell.

LASS, Roger (1997) Historical linguistics and language change, Cambridge: Cambridge University Press.

LOPE Blanch, Juan M. (1963) "El Atlas Lingüístico de la Península Ibérica”, Anuario de letras, 3, 233-252.

MARIÑO PAZ, Ramón (2017) Fonética e fonoloxía históricas da lingua galega, Vigo: Xerais.

McMaHON, April M. S. (1994) Understanding language change, Cambridge: Cambridge University Press.

MonTEAGUDO, Henrique (2020) "A individualidade do galego no pensamento lingüístico de Ricardo Carballo Calero", en Francisco Cidrás (ed.), Ricardo Carvalho Calero. As formas do compromiso, Santiago de Compostela: Universidade de Santiago de Compostela, 329360.

PÉREZ PASCUAL, José Ignacio (2016) Los primeros pasos de un largo caminar. Los comienzos del Atlas Lingüístico de la Península Ibérica, San Millán de la Cogolla: Cilengua.

PIÑEIRO, Ramón (2009) "Informe de Ramón Piñeiro á Real Academia Galega (edición e comentarios de Antón Santamarina)", Boletín da Real Academia Galega, 370, 7-17.

PORTO DAPENA, José Álvaro (1977) El gallego hablado en la comarca ferrolana, Santiago de Compostela: Universidade de Santiago de Compostela. 
Regueira, Xosé Luís (1996) "Os estudos de lingüística galega”, en Ramón LORENZO \& Rosario ÁlvaReZ (coords.), Homenaxe á profesora Pilar Vázquez Cuesta, Santiago de Compostela: Universidade de Santiago de Compostela, 47-67.

REgUeIRA, Xosé Luís (2008) “Os estudos de dialectoloxía galega desde 1967 á actualidade”, en Esther Corral díaz, Lydia Fontolra Suris \& Eduardo Moscoso Mato (eds.), a mi dizen quantos amigos ey. Homenaxe ao profesor Xosé Luís Couceiro, Santiago de Compostela: Universidade de Santiago de Compostela, 573-584.

RODRíGUEZ LORENZO, David (2012) "The diatopic development of the aspects of twentiethcentury Galician. A contrastive analysis of linguistic geography data", Dialectologia, Special Issue III, 143-156.

SÁNCHEZ REI, Xosé Manuel (2011) Lingua galega e variación dialectal, Ames: Laiovento.

Sanchis Guarner, Manuel, Lorenzo Rodríguez Castellano, Aníbal Otero \& Luís Filipe Lindley CINTRA (1961) “El Atlas Lingüístico de la Península Ibérica (ALPI). Trabajos, problemas y métodos", Boletim de Filologia, XX(1/22), 143-120.

SANTAMARINA, Antón (1975) “Aníbal Otero (1911-1974)”, Verba, 2, 7-11.

SANTAMARINA, Antón (1982) "Dialectoloxía galega: historia e resultados", en Dieter KREMER \& Ramón LORENZO (eds.), Tradición, actualidade e futuro do galego. Actas do Coloquio de Tréveris, Santiago de Compostela: Xunta de Galicia, 153-187.

SANTAMARINA, Antón (2005) "A lingüística galega entre 1960 e 1981", en 25 anos coa cultura galega. Vinte e cinco perspectivas para o século XXI, Santiago de Compostela: Sotelo Blanco, 189-215.

SOUSA, Xulio (2008) "Notas sobre o Atlas Lingüístico de la Península Ibérica”, en Mercedes BREA, Francisco Fernández Rei \& Xosé Luís Regueira (eds.), Cada palabra pesaba, cada palabra medía. Homenaxe a Antón Santamarina, Santiago de Compostela: Universidade de Santiago de Compostela, 299-306

SOUSA, Xulio (en prensa 1), "El Atlas Lingüístico de la Península Ibérica y el gallego del siglo XX". SOUSA, Xulio (en prensa 2) "La investigación geolingüística en Galicia: del siglo XX al siglo XXI”. TABOADA CID, Manuel (1976) El habla del valle de Verín, Santiago de Compostela: Universidade de Santiago de Compostela.

ZAMORA ViCEnTE, Alonso (1986 [1951]) “Geografía del seseo gallego", Filología, III/1-2: 84-95. Cito por la edición de Estudios de dialectología hispánica, Santiago de Compostela: Universidade de Santiago de Compostela, 1986, 1-10.

ZAMORA VICENTE, Alonso (1986 [1952]) "La frontera de la geada", en Homenaje a Fritz Krüger. Vol. 1, Mendoza: Universidad Nacional de Cuyo, 57-72. Cito por la edición de Estudios de 
Dialectologia. Special issue, IX (2021), 39-67.

ISSN: 2013-2247

dialectología hispánica, Santiago de Compostela: Universidade de Santiago de Compostela, 1986, 11-25.

ZAMORA VICENTE, Alonso (1986 [1953]), “De geografía dialectal: -ao, -án en gallego", Nueva Revista de Filología Hispánica, VII, 73-80. Cito por la edición de Estudios de dialectología hispánica, Santiago de Compostela: Universidade de Santiago de Compostela, 1986, 2734.

ZAMORA VICENTE, Alonso (1986 [1963]) "Los grupos -uit-, -oit- en gallego moderno: su repartición geográfica", Boletim de filologia, XXI, 57-68. Cito por la edición de Estudios de dialectología hispánica, Santiago de Compostela: Universidade de Santiago de Compostela, 1986, 35-44. 